\title{
Rigidly rotating dust solutions depending upon harmonic functions
}

\author{
Stefano Viaggiu \\ Dipartimento di Matematica, \\ Universitá di Roma "Tor Vergata", \\ Via della Ricerca Scientifica, 1, I-00133 Roma, Italy \\ E-mail: viaggiu@mat.uniroma2.it \\ (or: stefano.viaggiu@ax0rm1.roma1.infn.it)
}

August 4, 2021

\begin{abstract}
We write down the relevant field equations for a stationary axially symmetric rigidly rotating dust source in such a way that the general solution depends upon the solution of an elliptic equation and upon harmonic functions. Starting with the dipole Bonnor solution, we built an asymptotically flat solution with two curvature singularities on the rotational axis with diverging mass. Apart from the two point singularities on the axis, the metric is regular everywhere. Finally, we study a non-asymptotically flat solution with NUT charge and a massless ring singularity, but with a well-defined mass-energy expression.
\end{abstract}

PACS numbers: 04.20.-q, 04.20.Jb, 04.40.Nr

\section{Introduction}

The problem of building a physically admissible metric for an isolated rotating body is still long an unresolved problem [1, 9]. In fact, in order to obtain a physically reasonable source, many restrictions must be imposed (energy conditions, regularity, reasonable equation of state). In particular, Einstein's equations for a rotating body with a perfect fluid source seem not to be integrable. A remarkable exception is given by dust pressureless stationary axially symmetric spacetimes. In a remarkable paper [10], Winicour showed that Einstein's equations for a stationary axially symmetric dust source with differential rotation can be reduced to quadratures. These 
equations contain as a subclass the van Stockum one [11] of rigidly rotating

matter. The first asymptotically flat solution of the van Stockum class can be found in [12]. The author in [12] shows that the van Stockum class of solutions that are not cylindrically symmetric cannot exsist in the Newtonian theory. The solution in [12] has a curvature singularity with diverging mass. Further, the technique named "displace, cut, reflect" 13 to obtain rotating discs immersed in rotating dust must be noted. Unfortunately, this method generates distributional exotic matter on the $z=0$ plane.

In this paper, starting from the Lewis [14, 15] form of the metric, we write down the equations for stationary axially symmetric rigidly rotating spacetimes in a co-moving reference frame in such a way that the general solution depends upon the solution of an elliptic equation and upon harmonic functions. The class of solutions contains the van Stockum line element for a suitable choice of the harmonic function.

In this context, starting from the dipole Bonnor solution [12, we obtain an asymptotically flat solution with two curvature singularities on the rotation axis and showing simalar properties to the Bonnor solution.

In section 1 we derive the basic equations. In section 2 we present our solution. Section 3 collects some final remarks and conclusions. In the appendix we derive a non asymtotically flat solution with a NUT charge and a well-defined mass-energy expression.

\section{Basic Equations}

Our starting point is the Lewis [14 line element for a stationary axisymmetric space-time:

$$
d s^{2}=e^{v(\rho, z)}\left[d \rho^{2}+d z^{2}\right]+L(\rho, z) d \phi^{2}+2 m(\rho, z) d t d \phi-f(\rho, z) d t^{2},
$$

where $x^{4}=t$ is the time coordinate, $x^{1}=\rho$ is the radial coordinate in a cylindrical system, $x^{2}=z$ is the zenithal coordinate and $x^{3}=\phi$ is the azimuthal angular coordinate on the plane $z=0$. Also,

$$
t \in(-\infty, \infty), \rho \in(0, \infty), z \in(-\infty, \infty), \phi \in[0,2 \pi) .
$$

Further, the root square of the determinant of the 2-metric spanned by the Killing vectors $\partial_{t}, \partial_{\phi}$ is

$$
\sqrt{\left|\operatorname{det} g^{(2)}\right|}=\sqrt{f L+m^{2}}=W(\rho, z) .
$$

Expression (3) characterizes the measure of the area of the orbits of the isometry group. In the vacuum, the field equations for (11) imply that $W(\rho, z)$ 
is harmonic, i.e. $W_{, \alpha, \alpha}=0$, where subindices denote partial derivative and a summation with respect to $\alpha=\rho, z$ is implicit. Therefore, the function $W(\rho, z)$ can be chosen as a coordinate. Looking for regular solutions on the axis, the simplest assumption can be made by setting $W=\rho$. In this way, the van Stockum line element emerges by taking a dust source. However, this is not the most general choice. Thanks to the gauge freedom, we can take

$$
f L+m^{2}=\rho^{2} H(\rho, z),
$$

where $H(\rho, z)$ is a sufficiently regular function to be specified by the field equations.

We consider a perfect fluid $T_{\mu \nu}=(E+P) u_{\mu} u_{\nu}+P g_{\mu \nu}$, with $E$ being the mass-energy density,$P$ the hydrostatic pressure and $u_{\mu}$ the 4-velocity of the fluid. We consider a co-moving reference frame:

$$
u^{t}=\frac{1}{\sqrt{f}}, u^{\phi}=u^{\rho}=u^{z}=0
$$

Denoting with $R_{\mu \nu}$ the Ricci tensor, the relevant field equations are

$$
\begin{aligned}
& R_{z z}-R_{\rho \rho}=0, \\
& R_{\rho \rho}+R_{z z}=(P-E) e^{v}, \\
& R_{\rho z}=0, \\
& R_{\phi \phi}=\left[\frac{L}{2} T-T_{\phi \phi}\right], \\
& R_{t \phi}=\left[\frac{m}{2} T-T_{t \phi}\right], \\
& R_{t t}=-\left[\frac{f}{2} T+T_{t t}\right],
\end{aligned}
$$

where $T=3 P-E$. Equation (9) involves a second-order partial equation for $L(\rho, z)$, while (10) and (11) give second order equations for $m(\rho, z)$ and $f(\rho, z)$ respectively. Thanks to (4), equations (9)-(11) are not independent. Therefore, from (4), we can express $L(\rho, z)$ in terms of $(f, m, H)$. Putting this expression in (9) and using equations (10) and (11), we obtain the following compatibility equation:

$$
4 H P e^{v}=H_{, \alpha, \alpha}-\frac{H_{, \alpha}^{2}}{2 H}+\frac{2}{\rho} H_{, \rho} .
$$

In what follows we study dust solutions for which $P=0$. Setting $\rho^{2} H=F^{2}$, equation (12) becomes $F_{, \alpha, \alpha}=0$. Thus the compatibility condition for (9)(11) requires that $F(\rho, z)$ be a harmonic function. Conversely, with $F(\rho, z)$ 
no more harmonic, the line element (1) is appropriate to describe spacetimes with non-vanishing pressure $P$. For $F=\rho$ the van Stockum line element is regained together with the Papapetrou form of the metric [15]. Equations (6) and (8) are linear first-order equations involving $v_{, \rho}$ and $v_{, z}$ and they permit us to calculate $v_{, \rho}, v_{, z}$ in terms of $(f, m, F)$. By applying the integrability condition $\left(v_{, \rho, z}=v_{, z, \rho}\right)$ for the equations so obtained, we read

$$
f_{, z} F_{, \rho}=F_{, z} f_{, \rho}
$$

Finally, when expressions for $v_{, \rho}, v_{, z}$ are put in (7), we obtain

$$
F_{, z} f_{, z}=-f_{, \rho} F_{, \rho}
$$

Excluding the case $F=$ const (it can be see that this leads to the trivial solution $E=0$ ), we have $f=$ const. We naturally choose $f=1$. Therefore our system of equations is

$$
\begin{aligned}
& F_{, \alpha, \alpha}=0, \\
& m_{, \alpha, \alpha}-\frac{m_{, \alpha} F_{, \alpha}}{F}=0 \\
& E=\frac{e^{-v} m_{, \alpha}^{2}}{F^{2}}, \\
& v_{, \rho}=\frac{\left[m_{z}^{2} F_{, \rho}-m_{, \rho}^{2} F_{, \rho}+4 F F_{, z} F_{, z, \rho}-4 F F_{, \rho} F_{, z, z}-2 m_{, \rho} m_{, z} F_{, z}\right]}{2 F F_{, \alpha}^{2}}, \\
& v_{, z}=\frac{\left[4 F F_{, z} F_{, z, z}+4 F F_{, \rho} F_{, z, \rho}-F_{, z} m_{, z}^{2}+F_{, z} m_{, \rho}^{2}-2 m_{, \rho} m_{, z} F_{\rho}\right]}{2 F F_{, \alpha}^{2}}, \\
& L+m^{2}=F^{2} .
\end{aligned}
$$

First of all, for non-expanding spacetimes, the shear $q_{i k}=\frac{1}{2}\left[u_{i ; k}+u_{k ; i}\right]$ vanishes identically for (15)-(20), and therefore our system of equations describes rigidly rotating sources in a co-moving reference frame. When $F=\rho$, equation (16) is invariant under the transformation $z \rightarrow z+a$ ( $a$ a constant) and a solution can be expanded as $\sum_{i} m\left(\rho, z+a_{i}\right)$. Setting $F \neq \rho$, if $F(\rho, z), m(\rho, z)$ are solutions, then also $F(\rho, z+a), m(\rho, z+a)$ are, and thus the solutions cannot be expanded.

Note that we have identified $(\rho, z)$ with the radial and the zenithal coordinate respectively in a cylindrical coordinate system. According to this assumption, some conditions must be imposed. Firstly, by setting $E=0$, the metric must reduce to the standard flat expression $d s^{2}=d \rho^{2}+d z^{2}+\rho^{2} d \phi^{2}-d t^{2}$. Therefore, $\lim _{E \rightarrow 0} F=\rho$. Further, looking for regular spacetimes on the 
rotation axis , the norm of the space-like Killing vector $\partial_{\phi}$ must be vanishing (except at isolated points) at $\rho=0$, i.e. $\lim _{\rho \rightarrow 0} L=0$. Finally, for asymptotically flat spacetimes, at spatial infinity $F(\rho, z)$ looks as follows: $F=\rho+o(1)$.

\section{Generating an asymptotically flat solution}

Our starting point is the Bonnor dipole solution [12] $F=\rho, m=\frac{c \rho^{2}}{\left(\rho^{2}+z^{2}\right)^{\frac{3}{2}}}$. We can obtain a solution of (16) by taking the map $\rho \rightarrow F(\rho, z), z \rightarrow G(\rho, z)$, where $F=\rho\left(1+\frac{b c}{\left(\rho^{2}+z^{2}\right)}\right), G=z\left(1-\frac{b c}{\left(\rho^{2}+z^{2}\right)}\right)$ with $c \geq 0$, being $b$ a constant. Therefore we get the solution

$$
\begin{aligned}
& F=\rho\left(1+\frac{b c}{\left(\rho^{2}+z^{2}\right)}\right) \\
& m=\frac{c \rho^{2}\left[\rho^{2}+z^{2}+b c\right]^{2}}{\sqrt{\rho^{2}+z^{2}}\left[\left(\rho^{2}+z^{2}\right)^{2}+2 b c \rho^{2}+b^{2} c^{2}-2 b c z^{2}\right]^{\frac{3}{2}}}, \\
& v=\ln (\alpha)+\frac{c^{2} \rho^{2}}{8} \frac{\gamma\left(\rho^{2}+z^{2}+b c\right)^{2}}{\left[\left(\rho^{2}+z^{2}\right)^{2}+2 \rho^{2} b c+b^{2} c^{2}-2 z^{2} b c\right]}, \\
& E=\frac{c^{2} e^{-v} \beta \Delta}{\left[\left(\rho^{2}+z^{2}\right)^{2}+2 \rho^{2} b c+b^{2} c^{2}-2 z^{2} b c\right]^{4}}, \\
& \beta=\alpha\left(\rho^{2}+z^{2}\right)^{2}=\left(\rho^{2}+z^{2}\right)^{2}+c^{2} b^{2}-2 \rho^{2} c b+2 z^{2} c b, \\
& \gamma=\left(\rho^{2}-8 z^{2}\right)\left(\rho^{2}+z^{2}\right)^{2}+2 \rho^{4} c b+18 \rho^{2} z^{2} b c+\rho^{2} c^{2} b^{2}+16 z^{4} b c-8 z^{2} c^{2} b^{2}, \\
& \Delta=\left(\rho^{2}+4 z^{2}\right)\left(\rho^{2}+z^{2}\right)^{2}-8 z^{4} c b+4 z^{2} c^{2} b^{2}-6 \rho^{2} z^{2} c b+\rho^{2} c^{2} b^{2}+2 \rho^{4} c b .
\end{aligned}
$$

Solution (21) is asymptotically flat. Note that the map $\rho \rightarrow F(\rho, z), z \rightarrow$ $G(\rho, z)$ is not bijective, i.e. is not a diffeomorphism. Concerning the features of (21), they depend on the sign of the constant $b$.

For $b>0$, apart from $\rho=0, z= \pm \sqrt{b c}$, our solution is regular everywhere. At these two points, we have curvature singularities with properties close to the $\rho=0, z=0$ singularity of the dipole Bonnor solution (see [12]). In particular, the mass-energy diverges at these points. Otherwise, the energy density $E(\rho, z)$ is integrable. For $b=0$, we regain the Bonnor solution. Finally, for $b<0$, the two point singularities disappear, but emerges a curvature ring singularity for $z=0, \rho=\sqrt{|b| c}$ with diverging mass. Independently on the parameter $b$, at spatial infinity the metric reduces to the standard expression in asymptotical cylindrical coordinates, and so also by 
setting $c=0(E=0)$. Note that, because of the non-invertibility of the map between the Bonnor solution and solution (21), the curvature singularity at the origin of [12] is shifted in the two curvature singularities of (21) (for $b>0$ ) on the rotation axis. As a final consideration, it must be noted that there exists for (21) a finite non singular region about the origin. Thus, our solution could be matched, in principle, with some asymptotically flat vacuum solution. We do not enter in this discussion, but only mention this possibility.

\section{Conclusions and final remarks}

We have studied stationary axially symmetric rigidly rotating dust spacetimes in terms of harmonic functions. In [16] Bonnor found the general solution for charged dust with zero Lorentz force in terms of harmonic functions. However, the use of such kind of functions in [16] is different from the one in our paper. In fact, in our paper harmonic functions appear in equation (4) thanks to the gauge freedom, while in [16] the function $F(\rho, z)$ is chosen to be the cylindrical polar coordinate $\rho$. In the Bonnor paper, harmonic functions arise in order to obtain the most general solution for equation (16) with $F=\rho$. In this case, all the solutions of the equation $m_{, \alpha, \alpha}-\frac{1}{\rho} m_{, \rho}=0$ are given by taking a generic harmonic function $\eta(\rho, z)$, with $m=\rho \eta_{\rho}$. In a similar way, another harmonic function is introduced when charged dust comes in action. Therefore, a direct relation between does not exist between the harmonic function $F(\rho, z)$ and $\eta(\rho, z)$ of [16].

Also, the paper [17] must be noticed in which charged dust solutions are given in terms of Bessel functions of first and second kind and hyperbolic functions. Also in the paper [17] the condition $F=\rho$ is retained.

In this paper, section two, starting with the dipole Bonnor solution, we build a class of asymptotically flat solutions containing the Bonnor one as a subclass by a suitable choice of the functions $F(\rho, z), G(\rho, z)$. Obviously, it must be noted that not all the harmonic functions generate physically sensible solutions. For a physically sensible solution we mean a regular (apart from isolated singularities) asymptotically flat solution. Generally, it is a simple matter to verify that, if $F(\rho, z)=\rho, m(\rho, z)$ is a regular differentiable solution for (16), then also $m(F(\rho, z), G(\rho, z))$ is a solution for (16) with $F(\rho, z)$ harmonic being $G(\rho, z)$ the harmonic conjugate to $F(\rho, z)$ i.e. $F_{\rho}=G_{z}, F_{z}=-G_{\rho}$. Further, in order to generate a new asymptotically flat and regular solution on the axis starting with a seed solution with these two properties, we must build a non-bijective (not a diffeomorphism) map 
$\rho \rightarrow F(\rho, z), z \rightarrow G(\rho, z)$ such that $\lim _{E \rightarrow 0} F=\rho, \lim _{E \rightarrow 0} G=z$, and $\lim _{\rho \rightarrow 0} L=\lim _{\rho \rightarrow 0} F=0$ (apart from isolated points) and such that at spatial infinity the functions $F(\rho, z), G(\rho, z)$ look as follows: $F=\rho+o(1), G=$ $z+o(1)$. Concerning isolated singularities, no general conclusions can be made. The functions $F(\rho, z), G(\rho, z)$ of section two satisfy all the conditions mentioned above.

Finally, in the appendix we present a non-asymptotically flat solution not obtained from a seed solution with the technique discussed above and therefore it represents an ad hoc solution. In particular, it is possible to build ad hoc solutions for (16) by setting $F(\rho, z)=\rho\left(1+\frac{c}{\rho^{2}+z^{2}}\right)$ (with $c$ a constant) and $m(\rho, z)$ a homogeneous function such that $m_{, \alpha, \alpha}-\frac{m_{, \rho}}{\rho}=0$.

\section{Appendix}

We consider the following solution:

$$
\begin{aligned}
& F=\rho\left[1+\frac{c}{\rho^{2}+z^{2}}\right] \quad, \quad m=c\left[\frac{z}{\sqrt{\rho^{2}+z^{2}}}-1\right], \\
& v=\left[1+\frac{c}{16}\right] \ln \left[\rho^{4}-2 c \rho^{2}+2 \rho^{2} z^{2}+c^{2}+z^{4}+2 c z^{2}\right] \\
& -\frac{c}{8} \ln \left[c+\rho^{2}+z^{2}\right]-2 \ln \left[\rho^{2}+z^{2}\right], E=\frac{c^{2} e^{-v}}{\left[c+\rho^{2}+z^{2}\right]^{2}},
\end{aligned}
$$

with $c$ a constant. When $c>0$, the solution (22) has a curvature ring singularity when $(\rho, z)=(\sqrt{c}, 0)$ The axis is regular for $z>0$, while it shows a conical (no curvature) singularity when $z \leq 0$. Further, for $z<0$ there is a region where closed time-like curves (CTC) appear resulting in a vioaltion of causality. However, it is possible to take a simple coordinate transformation found in [18], i.e. $\tau=t+2 c \phi$ giving the whole rotational axis free of conical singularities. Unfortunately, we are forced to introduce a periodic time coordinate $\tau$ and therefore once again CTC appear. Therefore, the problem of violation of causality cannot be avoided with an opportune coordinate transformation.

The spacetime asymptotically reads the expression appropriate for asymptotic NUT metrics [19, 20] with NUT charge $q$ given by $c=2 q$. We can estimate the mass inside an infinite cylinder of radius $R$ by means of the integral

$$
M(R)=\int_{-\infty}^{\infty} d z \int_{0}^{R} d \rho \int_{0}^{2 \pi} E F e^{v} d \phi
$$


The integral (23) is well defined everywhere. Thus, for the mass we get the formula

$$
M(R)=2 \pi^{2} c^{2}\left[1+R-\sqrt{R^{2}+1}\right] .
$$

Because of the non-asymptotical flatness, the solution (22) is not interesting in an astrophysical context. However, the natural arena for this solution is in the extra relativistic context given by non-Abelian gauge theories or in the low energy string theory [21, 22] where, in order to obtain supersymmetries, NUT charge comes in action.

\section{References}

[1] Neugebauer G and Meinel R 1993 Astrophys. J. 414 L97

[2] Senovilla J M M 1987 Class. Quantum Grav. 4 L 115

[3] Senovilla J M M 1992 it Class. Quantum Grav. 9 L 167

[4] Wahlquist M D 1968 Phys. Rev. 1721291

[5] Kramer D 1985 Class. Quantum Grav. 2 L 135

[6] Stephani M 1988 J. Math. Phys. 291650

[7] Stewart J M and Ellis GFR 1968 J. Math. Phys. 91072

[8] Herlt E 1988 it Gen. Rel. Grav. 20635

[9] Lukacs B et alt. 1983 Gen. Rel. Grav. 15567

[10] Winicour J 1975 J. Math. Phys. 161805

[11] Stockum V 1937 Proc. Roy. Soc. Eddim. 57135

[12] Bonnor W B 1977 J. Phys. A: Math. Gen. 101673

[13] Vogt D and Letelier P S 2006 Preprint astro-ph/0611428 (To appear in IJMPD)

[14] Lewis T 1932 Proc. Roy. Soc. Lond. 136176

[15] Papapetrou V A 1953 Ann. Phys., Lpz 612

[16] Bonnor W B 1980 J. Phys. A: Math. Gen. 133465

[17] Georgiou A 2001 Proc. Royal Soc.: Math. Phys. Sci. 4571153 
[18] Misner CW 1963 J. Math. Phys. 4924

[19] Newman E, Tamburino L and Unti T 1963 J. Math. Phys. 4915

[20] Dadhich N and Turakulov Z Y 2002 Class. Quantum Grav. 192765

[21] Radu E 2003 Phys. Rev. D 67084030

[22] Johnson C V and Myers R C 1994 Phys. Rev. D 506512 